\title{
A New Tensile Test for Aluminum Alloys in the Mushy State: Experimental Method and Numerical Modeling
}

\author{
V. MATHIER, P.-D. GRASSO, and M. RAPPAZ \\ A fairly simple experimental setup has been designed for testing the resistance of the mushy zone \\ of alloys during solidification under tensile conditions. It has been used to study the effect of \\ coalescence among the solid grains at a late stage of solidification. The experimental approach \\ involves both tensile-strength measurements and scanning electron microscope (SEM) obser- \\ vations of fracture surfaces. Complementary information can be obtained by numerical mod- \\ eling of this solidification process. The latter takes into account heat flow in the sample, \\ rheology of the mushy alloy, liquid feeding, and porosity formation. All of the available \\ information indicates that the transition from a granular mushy alloy to a coalesced solid- \\ skeleton behavior starts for a solid fraction of approximately 92 pct.
}

DOI: $10.1007 / \mathrm{s} 11661-008-9529-\mathrm{y}$

(C) The Minerals, Metals \& Materials Society and ASM International 2008

\section{INTRODUCTION}

HoT tearing is a severe defect that occurs during solidification processes. It initiates in the mushy zone due to the interplay between two main mechanisms: deformation of the partially coherent solid and lack of interdendritic liquid feeding. ${ }^{[1-4]}$ While the latter aspect can be approached with Darcy-type models, deformation of the solid skeleton is more complex to handle because it depends on the coherency state, i.e., on how strains are transmitted at the microscopic level. The transition from continuous liquid films to a fully coalesced solid is gradual; in the critical region of the mushy zone where coalescence is only partially reached, strains are localized at remaining wet grain boundaries. This transition has been studied recently on the basis of thermodynamic and statistical approaches, ${ }^{[5-7]}$ but experimental observations are still scarce.

There exist several classical tests to characterize the sensitivity of alloys to hot tearing, mainly the I-beam or dog-bone tests ${ }^{[8-9]}$ and the ring or conical-mold tests. ${ }^{[10]}$ In most of these tests, one measures the amount of cracks formed under given conditions as a function of the alloy concentration. In addition to measurements performed during remelting of alloys, a few systems have been used to measure the strength developed by mushy zones during solidification. Ackerman et al. ${ }^{[11]}$ designed a system made of two water-cooled copper cylinders, which could be directly plunged into a melt. After formation of a given solidified thickness around

V. MATHIER, Development Engineer, is with the Computational Materials Laboratory, Ecole Polytechnique Fédérale de Lausanne, and the Calcom-ESI SA, Parc Scientifique, EPFL, CH-1015 Lausanne, Switzerland. Contact e-mail: vincent.mathier@esi-group.com P.-D. GRASSO, Engineer, and M. RAPPAZ, Professor, are with the Computational Materials Laboratory, Institute of Materials Science, School of Engineering, Ecole Polytechnique Fédérale de Lausanne, CH-1015 Lausanne, Switzerland.

Manuscript submitted November 9, 2007

Article published online April 16, 2008 the two halves, they can be moved apart while the force is recorded. This device has the advantage of accurately reproducing the conditions of hot tearing (straining is indeed initiated from the solid part) but has two drawbacks: safety and difficult interpretation as the stress has to be integrated over a solid-fraction gradient. Several devices similar to tensile-test experiments (and adapted to mushy alloys) have been used to either measure stresses developed upon restrained contraction of the solid or stress-strain curves upon imposed displacement. They are reviewed in more detail in the literature. ${ }^{[1]}$

The onset of coalescence is important when considering hot tearing in low- concentration alloys, because it corresponds to the transition between a continuous network of liquid films and a fully coalesced solid skeleton through the formation of intergranular solid bridges. ${ }^{[12]}$ Intragranular coalescence, i.e., bridging of dendrite arms belonging to the same grain, occurs at a fairly high temperature. ${ }^{[5]}$ This intragranular bridging mechanically consolidates the grains, which become the elementary unit for mechanical deformation and thermal contraction. Coalescence at low-angle grain boundaries occurs next, thus initiating the first clusters of grains and increasing the apparent grain size. ${ }^{[6-13]}$ This occurs at a stage of solidification when feeding starts to be difficult, i.e., at the beginning of the hot-cracking sensitive zone. ${ }^{[7-13]}$ Coalescence at large misorientation grain boundaries occurs deeper in the mushy zone and corresponds to the formation of a fully coalesced solid. Evaluating the temperatures at which these phenomena appear, and thus determining the critical range of solid fraction where a coherent solid forms, is then fundamental to advance the study of hot tearing. Using the rig test with fixed jaws, ${ }^{[14]} \mathrm{Ju}$ determined that coalescence in Al-1 and -2 wt pct $\mathrm{Cu}$ reached significant levels at solid fractions in the range of 90 to 97 pct. $^{[15]}$ Using a tensile test with local remelting followed by partial solidification, Ludwig found for $\mathrm{Al}-2$ and -4 wt pet $\mathrm{Cu}$ similar values in the range between 94 and 96 pct of solid fraction. ${ }^{[16]}$ 
With the objective of more precisely describing the process of coalescence and in which way this phenomenon takes place, a new experiment was designed. Similar to several other fairly complex devices, the present setup allows for a measurement of the tensile strength of the mushy zone during solidification. This has the advantage of using a fairly simple design and does not involve any interaction with a free (oxidized) surface. This experimental approach is complemented with numerical modeling in order to assess the development of a coherent solid network in an Al-1 wt pet $\mathrm{Cu}$ alloy.

\section{EXPERIMENTAL METHOD}

The experimental setup is designed to solidify the alloy while applying strain to the mushy zone at various fractions of solid. The solidifying alloy is contained in a mold such that the mushy zone is not in direct contact with air, thus preventing oxidation. A schematic diagram of this hot-tearing test with the indication of the main dimensions is shown in Figure 1. The setup consists of a cylindrical stainless-steel mold with a reduced cross section (neck) at midheight. This geometry was selected to concentrate the strains applied to the mushy alloy. The mold is made of two parts that may be separated, with the separation plane being at the level of the lowest part of the neck. The upper half is attached to the mobile jaw of a standard tensile-test machine, and the lower half is fixed to an immobile copper plate. An annular guide is used to align both halves prior to the test. This mold also allows for a thermocouple to be inserted in the separation plane to record the temperature at the bottom of the neck close to the mold. The lateral faces of the mold are wrapped in 10-mm-thick ceramic wool. Under such conditions, directional solidification of the Al-1 wt pet $\mathrm{Cu}$-alloy sample is achieved in a low-thermal gradient. The latter alloy was selected for this study because of its high hottearing tendency.

The experimental procedure was as follows. After melting the alloy in a furnace, the melt was poured into the preheated mold, which was then put back in the furnace. Once the temperature became uniform, the setup was fixed to the copper plate and attached to the jaw of the tensile-test machine (MFL $250 \mathrm{kN}$ ). Once the desired temperature, $T_{\text {tear }}$, was reached at the bottom of the neck (i.e., as measured by the thermocouple), a constant displacement rate of $3.3 \mathrm{~mm} \mathrm{~s}^{-1}$ was applied to the upper part of the mold, and the reaction force was measured using a $500 \mathrm{~N}$ load cell.

This setup was used to study the coalescence of the primary phase in an Al-1 wt pet $\mathrm{Cu}$ alloy. With this composition, the amount of eutectic is very small and forms at a time when most of the primary phase has

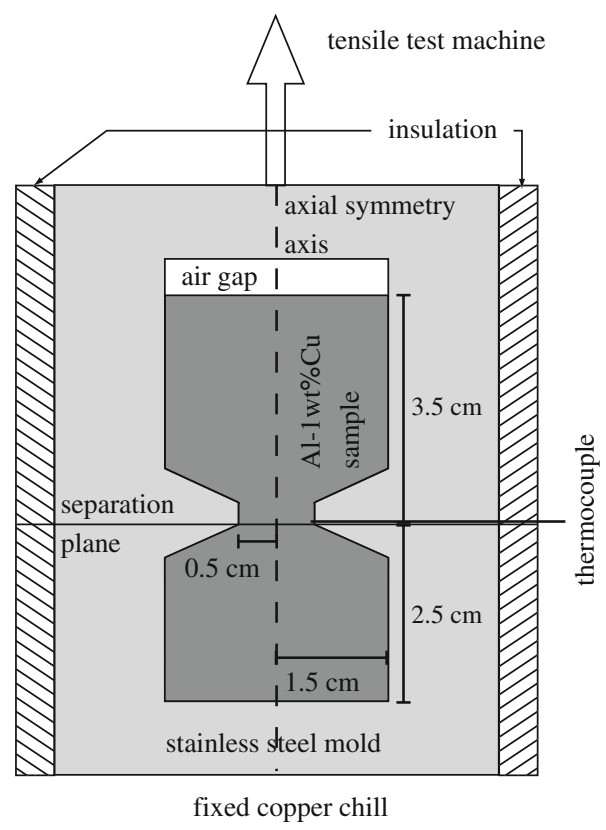

Fig. 1-Schematic representation of the tearing experiment: a stainless steel mold, which can be separated in two halves, is insulated on the side and fixed to a copper chill at the bottom. The dogboneshaped Al-1 wt pet $\mathrm{Cu}$ sample that is cast is thus solidified from the bottom and experiences strain due to the displacement rate imposed to the top half of the mold by the tensile test machine.

coalesced already (as could be checked by metallographic observations). In order to avoid the formation of columnar grains, the alloy was inoculated using AlTiB 1/5 from Anglo Blackwells, Widnes, UK (approximately $0.1 \mathrm{wt}$ pet). The chemical composition of a torn specimen was done by Alcan (Chippis, $\mathrm{CH}$ ), using spectrometric analysis (Table I). This clearly shows that no detectable reaction with the mold wall occurred during the test (i.e., low amount of iron).

\section{NUMERICAL MODEL}

\section{A. Method}

In order to simulate the tensile test described previously, a semicoupled approach was selected. Details about this method can be found in the literature ${ }^{[17,18]}$ and we shall thus only summarize its most important features here (see Table II for symbols definition). First of all, the thermal field in the mold and the sample is calculated using an appropriate finite-element package (CALCOSOFT3D* in

*CALCOSOFT3D is a trademark of Calcom-ESI, Lausanne, CH.

the present case). The solid-fraction field can be deduced

Table I. Composition of the Al-I Wt Pct Cu Inoculated Alloy after the Tearing Test in Weight Percent

\begin{tabular}{lcccccccccc}
\hline $\mathrm{Si}$ & $\mathrm{Fe}$ & $\mathrm{Cu}$ & $\mathrm{Mn}$ & $\mathrm{Mg}$ & $\mathrm{Cr}$ & $\mathrm{Ni}$ & $\mathrm{Zn}$ & $\mathrm{Ti}$ & $\mathrm{V}$ \\
\hline $0.008 \pm 0.001$ & $0.021 \pm 0.002$ & $0.95 \pm 0.03$ & $<0.001$ & $<0.003$ & $0.0022 \pm 0.002$ & $<0.001$ & $<0.002$ & 0.0045 & $<0.001$ \\
\hline
\end{tabular}


Table II. List of Symbols

\begin{tabular}{|c|c|c|}
\hline Symbol & Meaning & Unit \\
\hline$\alpha$ & $\begin{array}{l}\text { rate of evolution of cohesion at } \\
\text { low strain }\end{array}$ & $(-)$ \\
\hline$A_{2}, A_{3}$ & softening functions & $(-)$ \\
\hline$C$ & $\begin{array}{l}\text { internal variable describing the } \\
\text { cohesion of the mushy alloy }\end{array}$ & $(-)$ \\
\hline$C^{*}$ & saturation value of the cohesion & $(-)$ \\
\hline$\dot{\boldsymbol{\varepsilon}}^{v p}$ & viscoplastic strain-rate tensor & $\left(\mathrm{s}^{-1}\right)$ \\
\hline$\dot{\varepsilon}_{0}$ & thermally activated coefficient & $\left(\mathrm{s}^{-1}\right)$ \\
\hline$\dot{\varepsilon}_{c r}$ & $\begin{array}{l}\text { creep (constant volume) charac- } \\
\text { teristic strain rate }\end{array}$ & $\left(\mathrm{s}^{-1}\right)$ \\
\hline$\dot{\varepsilon}_{s w}$ & $\begin{array}{l}\text { swelling (volumetric) } \\
\text { characteristic strain rate }\end{array}$ & $\left(\mathrm{s}^{-1}\right)$ \\
\hline$\phi$ & grain size & $(\mathrm{m})$ \\
\hline g & gravity vector & $(-)$ \\
\hline$g_{s}$ & solid fraction & $(-)$ \\
\hline$g_{s}^{\mathrm{coh}}$ & $\begin{array}{l}\text { coherency point (above this solid } \\
\text { fraction, strains are } \\
\text { transmitted to the mushy alloy) }\end{array}$ & $(-)$ \\
\hline$g_{s}^{\text {coal }}$ & $\begin{array}{l}\text { coalescence point (above this } \\
\text { solid fraction, the alloy } \\
\text { behaves as a continuous solid) }\end{array}$ & $(-)$ \\
\hline I & unit tensor & $(-)$ \\
\hline$K$ & permeability of the solid skeleton & $\left(\mathrm{m}^{2}\right)$ \\
\hline$l$ & $\begin{array}{l}\text { subscript indicating the liquid } \\
\text { phase }\end{array}$ & $(-)$ \\
\hline$\mu$ & viscosity of the liquid phase & (Pa s) \\
\hline$n$ & exponent of the power-law creep & $(-)$ \\
\hline$P_{s}$ & $\begin{array}{l}\text { pressure in the solid phase (first } \\
\text { invariant of the stress tensor) }\end{array}$ & $(\mathrm{Pa})$ \\
\hline$p_{l}$ & pressure in the liquid phase & $(\mathrm{Pa})$ \\
\hline$\rho_{l}$ & density of the liquid phase & $\left(\mathrm{kg} \mathrm{m}^{-3}\right)$ \\
\hline $\bar{\rho}$ & $\begin{array}{l}\text { average density of the mixture of } \\
\text { phases }\end{array}$ & $\left(\mathrm{kg} \mathrm{m}^{-3}\right)$ \\
\hline$T_{\text {tear }}$ & $\begin{array}{l}\text { temperature recorded by the } \\
\text { thermocouple when the tensile } \\
\text { test is started }\end{array}$ & $\left({ }^{\circ} \mathrm{C}\right)$ \\
\hline$T$ & temperature & $\left({ }^{\circ} \mathrm{C}\right)$ \\
\hline$\sigma_{M}$ & $\begin{array}{l}\text { Von Mises stress (second } \\
\text { invariant of the stress tensor) }\end{array}$ & $(\mathrm{Pa})$ \\
\hline$\sigma$ & total stress & $(\mathrm{Pa})$ \\
\hline $\boldsymbol{\sigma}_{\text {eff }}$ & effective stress & $(\mathrm{Pa})$ \\
\hline$s$ & $\begin{array}{l}\text { subscript indicating the solid } \\
\text { phase }\end{array}$ & $(-)$ \\
\hline$s_{0}$ & $\begin{array}{l}\text { characteristic stress resistance of } \\
\text { the solid phase }\end{array}$ & $(\mathrm{Pa})$ \\
\hline $\mathbf{v}_{s}$ & solid velocity & $\left(\mathrm{m} \mathrm{s}^{-1}\right)$ \\
\hline$X$ & stress triaxiality & $(-)$ \\
\hline
\end{tabular}

from the solidification path, $g_{s}(T)$, which is input by the user.

Once a good description of the thermal field is available, the problem of mass and momentum conservation can be addressed. In general, the momentumconservation equation can be written as follows: ${ }^{[19]}$

$$
\nabla \cdot \boldsymbol{\sigma}_{\text {eff }}+\bar{\rho} \mathbf{g}=\nabla p_{l}
$$

where $\bar{\rho}=g_{s} \rho_{s}+g_{l} \rho_{l}$ is the two-phase averaged density ( $g$ indicates volume fraction, $\rho$ density, and the $s$ and $l$ subscripts denote the solid and liquid phase, respectively); $p_{l}$ is the pressure in the liquid phase; $\mathbf{g}$ is the gravity vector; and the effective stress, $\boldsymbol{\sigma}_{\text {eff }}=\boldsymbol{\sigma}+p_{l} \mathbf{I}$, can be defined from the total (measured) stress, $\boldsymbol{\sigma}$. Within the framework of the present approach, the liquid pressure and its variation remain small (compared to the stress and its gradient), so it is reasonable to neglect its effect on the mechanical problem. The more usual form of the momentum conservation is thus solved as follows to obtain the strain and strain-rate fields in the sample: ${ }^{[20]}$

$$
\nabla \cdot \boldsymbol{\sigma}+\rho \mathbf{g}=0
$$

The resolution of this problem is carried out using the thermal and solid-fraction field previously calculated as input. The rheology of the material is described using the model of Ludwig et al., ${ }^{[16]}$ which was implemented in ABAQUS**. This model is expressed in the following

**ABAQUS is a trademark of Abaqus Inc., Pawtucket, RI.

form for the coherent mushy zone (i.e., where $\left.g_{s}>g_{s}^{\mathrm{coh}}\right)$ :

$$
\begin{aligned}
\dot{\boldsymbol{\varepsilon}}^{v p} & =\frac{\dot{\varepsilon}_{0}}{\left(C s_{0}\right)^{n}}\left(A_{2} P_{s}^{2}+A_{3} \sigma_{M}^{2}\right)^{\frac{n-1}{2}}\left(-\frac{A_{2}}{3} P_{s} \mathbf{I}+\frac{3}{2} A_{3} \mathbf{S}\right) \\
& =\dot{\varepsilon}_{s w} \mathbf{I}+\frac{3}{2} \frac{\dot{\varepsilon}_{c r}}{\sigma_{M}} \mathbf{S}
\end{aligned}
$$

where $\dot{\boldsymbol{\varepsilon}}^{v p}=\frac{1}{2}\left(\nabla \mathbf{v}_{s}+\nabla \mathbf{v}_{s}^{T}\right)$ is the viscoplastic strain-rate tensor; $\dot{\varepsilon}_{S w}$ and $\dot{\varepsilon}_{c r}$ are the volumetric (swelling in ABAQUS) and deviatoric (creep in ABAQUS) equivalent strain rates, respectively; $P_{S}=-\frac{1}{3} \operatorname{tr}(\boldsymbol{\sigma})$ is the pressure in the solid; $\mathbf{S}=\boldsymbol{\sigma}+P_{s} \mathbf{I}$ is the deviatoricstress tensor; and $\sigma_{M}$, its second invariant $\left(\sigma_{M}^{2}=\frac{3}{2} \mathbf{S}: \mathbf{S}\right)$. The term $C$ is the cohesion internal variable, which varies from zero (freely floating solid grains in liquid) to one (dense solid or fully coalesced granular skeleton). Additional closing relations are as follows: ${ }^{[16]}$

$$
\begin{aligned}
& \dot{\varepsilon}_{0}=A \exp \left(-\frac{Q}{\mathrm{R} T}\right) \\
& A_{2}=\frac{9}{4}\left\{n\left[\left(1-g_{s}\right)^{-\frac{1}{n}}-1\right]\right\}^{\frac{-2 n}{n+1}} \\
& A_{3}=\left[1+\frac{2}{3}\left(1-g_{s}\right)\right]\left(g_{s}\right)^{\frac{-2 n}{n+1}}
\end{aligned}
$$

The model is completed by the following evolution equation for the internal variable $C::^{[16]}$

$$
\frac{d C}{d t}=\alpha\left(1-\frac{C}{C^{*}}\right) \sqrt{\frac{2}{3} \dot{\boldsymbol{\varepsilon}}^{v p}: \dot{\boldsymbol{\varepsilon}}^{v p}}
$$

Introducing the triaxiality, $X=\frac{P_{s}}{\sigma_{M}}$ ( $<0$ in tension), the following relations are adopted: $:^{[16]}$

$$
\begin{aligned}
& \alpha\left(g_{s}, X \geq 0\right)=\alpha_{0}+\alpha_{1} \frac{g_{s}^{\frac{1}{3}}}{1-g_{s}^{\frac{1}{3}}} \\
& \alpha\left(g_{s}, X<0\right)=\alpha_{0}+\alpha_{1} \frac{g_{s}^{\frac{1}{3}}}{1-g_{s}^{\frac{1}{3}}} \exp \left[k\left(g_{s}-g_{s}^{\text {coal }}\right)\right] \\
& C^{*}\left(g_{s}, X>2\right)=1 \\
& C^{*}\left(g_{s}, X \leq 2\right)=1-\left(1-g_{s}\right)^{p}
\end{aligned}
$$


These expressions take into account the dependence of the mushy-zone behavior on the stress state. Moreover, the effect of coalescence of the grains at high solid fractions is introduced above a critical fraction, $g_{s}^{\text {coal }}$, of typically 96 pct. The materials parameters (activation energy, $Q$; constants, $A, k, \alpha_{0}$, and $\alpha_{1}$; and exponents, $p$ and $n$ ) that appear in this model were determined by experimental identification. ${ }^{[16,21,22]}$

In the case of the fully solid material $\left(g_{s}=1, C=1\right)$, the following model takes a more classical form:

$$
\dot{\boldsymbol{\varepsilon}}^{v p}=\frac{3}{2} \frac{\dot{\varepsilon}_{c r}}{\sigma_{M}} \mathbf{S} \quad \text { with } \quad \dot{\varepsilon}_{c r}=\frac{\dot{\varepsilon}_{0}}{s_{0}^{n}} \sigma_{M}^{n}
$$

Having solved the mechanical problem, the velocity field of the solid, $\mathbf{v}_{\mathrm{s}}$, is known, and the mass-conservation equation for the liquid pressure may be solved. The latter step is performed using the $\mathrm{PROCAST}^{\dagger}$ porosity

${ }^{\dagger}$ PROCAST is a trademark of ESI-Group, Paris, FR.

module, in which the influence of strain is taken into account, $\nabla \cdot \mathbf{v}_{s}=\dot{\varepsilon}_{s w}$. The following form of the massconservation equation is thus solved: ${ }^{[19]}$

$$
\frac{\partial \bar{\rho}}{\partial t}+\nabla \cdot\left(\bar{\rho} \mathbf{v}_{s}\right)-\nabla\left(\frac{\rho_{l} K}{\mu}\left(\nabla p_{l}-\rho_{l} \mathbf{g}\right)\right)=0
$$

where $\mu$ is the viscosity of the liquid phase, and $K=\frac{\left(1-g_{s}\right)^{3}}{g_{s}^{2}} \frac{\phi^{2}}{180}$ is the permeability of the solid skeleton and depends on the grain size, $\phi$. The formation of porosity due to the presence of hydrogen dissolved in the alloy can also be predicted using the model described in the literature. ${ }^{[23]}$ In summary, the flow chart presented in Figure 2 was followed to obtain a description of the preceding experiment using a continuum-scale approach.

Finally, please note that the solid fraction takes into account the effect of strain in both the mechanical and porosity calculations. For these cases, the solidification path, $g_{s}(T)$, which is provided as input and used directly in the heat-flow simulation, is corrected according to the following equation:

$$
g_{s}^{\varepsilon}(T)=g_{s}^{0}(T)\left(1-\int \operatorname{tr}\left(\underline{\boldsymbol{\varepsilon}}^{v p}\right) d t\right)
$$

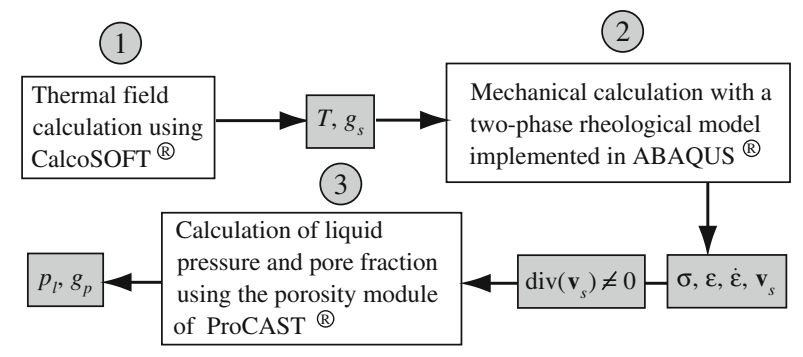

Fig. 2-Flow chart for the numerical simulation of the tearing experiment. where $g_{s}^{\varepsilon}(T)$ is the volume fraction of solid under deformation conditions, and $g_{s}^{0}(T)$ is the usual solidification path without applied strain (in what follows, we shall use the notation $g_{s}$ to denote $g_{s}^{\varepsilon}(T)$, while $g_{s}^{0}(T)$ will be used only when the thermal solid fraction is specifically referred to). The latter path can be deduced from a microsegregation model or from thermal analyses in the absence of deformation (see Section B).

\section{B. Parameters}

Having recalled the most important steps involved in the numerical modeling of the present experiment, the actual parameters that were used in this study will now be summarized. First of all, the thermal-boundary conditions are described in Figure 3. Please note that the axisymmetry of the problem is taken into account and that the mold was included in the mesh at this stage. The symmetry axis, interface heat-transfer condition, and heat exchange at free surfaces are modeled as summarized in Table III. This table also contains the materials parameters that were used.

The solidification path, $g_{s}^{0}(T)$, of the Al-1 wt pet $\mathrm{Cu}$ alloy was obtained from thermal measurements available in the literature. ${ }^{[24]}$ As shown in Figure 5, solidification starts at $653{ }^{\circ} \mathrm{C}$ and is terminated at $560{ }^{\circ} \mathrm{C}$. This last value is slightly higher than the eutectic temperature $\left(548{ }^{\circ} \mathrm{C}\right)$ and clearly shows that coherency is reached primarily by coalescence of the primary phase in this alloy. A computation of $g_{s}^{0}(T)$ based on a ScheilGulliver approximation (i.e., no back diffusion) would give only 1.5 pct of eutectic. The measured curve, $g_{s}^{0}(T)$,

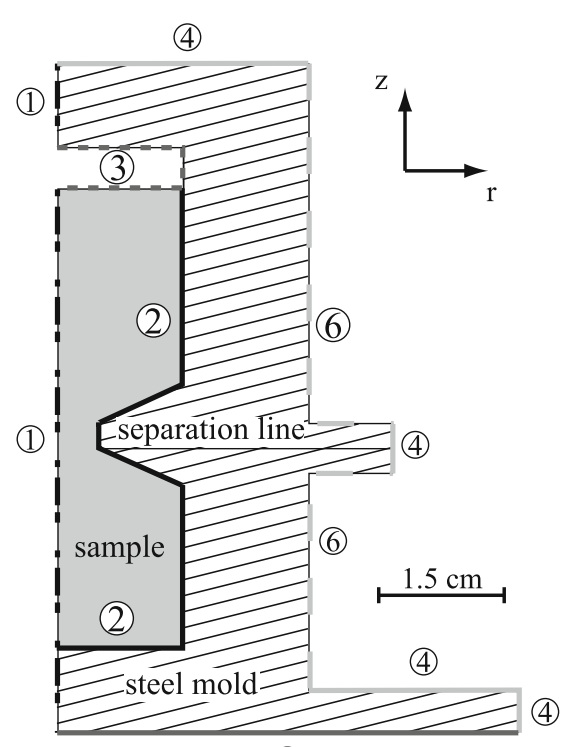

(5)

$$
\begin{aligned}
& \text { (1) --- symmetry (4) }- \text { ambient air } \\
& \text { (2) }- \text { mold - sample (5) }- \text { copper chill } \\
& \text { interface } \\
& \text { (3) }=-=\text { air gap }
\end{aligned}
$$

Fig. 3- Geometry and thermal boundary conditions used to simulate the tearing test. 
Table III. Summary of the Parameters Involved in the Thermal Simulation

\begin{tabular}{|c|c|}
\hline $\begin{array}{l}\text { Thermal Boundary Condition as Defined in Figure } 3 \\
\text { 1: axial symmetry } \\
\text { 2: mold-sample interface } \\
\text { 3: air gap } \\
\text { 4: ambient air } \\
\text { 5: copper chill } \\
\text { 6: insulating wool }\end{array}$ & $\begin{array}{l}\mathrm{h} \text { in } \mathrm{Wm}^{-1} \mathrm{~K}^{-1} ; \mathrm{T} \text { in }{ }^{\circ} \mathrm{C} \\
\text { zero heat flux } \\
\text { interface transfer: } h_{2}=850 \\
\text { convective transfer: } h_{3}=20, T_{\text {ext }, 3}=700 \\
\text { convective transfer: } h_{4}=20, T_{\text {ext, } 4}=25 \\
\text { convective transfer: } h_{5}=120, T_{\text {ext }, 5}=25 \\
\text { convective transfer: } h_{6}=1, T_{\text {ext, } 6}=25\end{array}$ \\
\hline $\begin{array}{l}\text { Properties of the Al-1 Wt Pct Cu Sample } \\
\text { Thermal conductivity } \\
\text { Heat capacity } \\
\text { Latent heat }\end{array}$ & $\begin{array}{l}\kappa=170 \mathrm{Wm}^{-1} \mathrm{~K}^{-1} \\
\rho C_{p}=3.2 \cdot 10^{6} \mathrm{Jm}^{-3} \mathrm{~K}^{-1} \\
\rho L=9 \cdot 10^{8} \mathrm{Jm}^{-3}\end{array}$ \\
\hline $\begin{array}{l}\text { Properties of the Stainless Steel Mold } \\
\text { Thermal conductivity } \\
\text { Heat capacity }\end{array}$ & $\begin{array}{l}\kappa=40 \mathrm{Wm}^{-1} \mathrm{~K}^{-1} \\
\rho C_{p}=5 \cdot 10^{6} \mathrm{Jm}^{-3} \mathrm{~K}^{-1}\end{array}$ \\
\hline
\end{tabular}

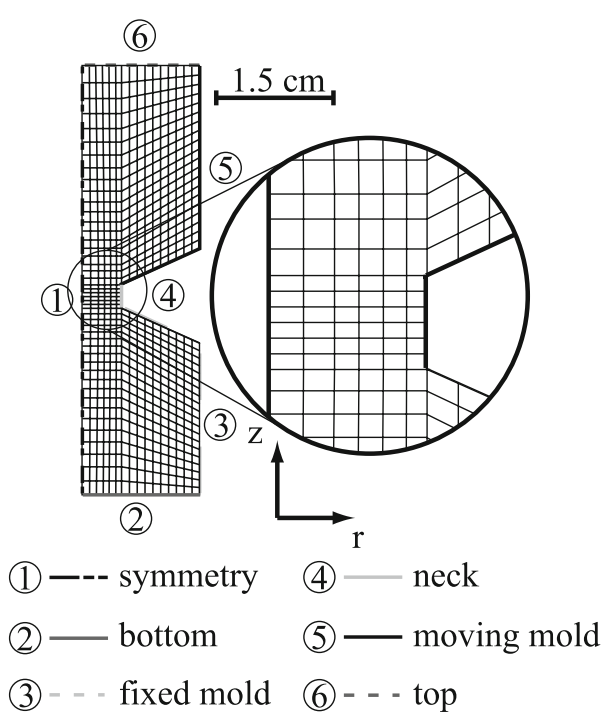

Fig. 4-Mechanical boundary conditions applied to the sample and mesh.

is compared in Figure 5 to the lever rule and ScheilGulliver models.

As far as the mechanical problem is concerned, the boundary conditions are quite straightforward. Please note that the mold, assumed to be rigid, was not included in these calculations. The axisymmetry of the problem was used as suggested in Figure 4. Again, the boundary conditions and material properties are summarized in Table IV.

Thermal contraction of the sample was found to be negligible when compared to the strain rate applied by the tensile-test machine. The rheology of the alloy was described using the parameters from the literature ${ }^{[16]}$ as summarized in Table V. Although these parameters were measured for Al-2 wt pet $\mathrm{Cu}$ and $\mathrm{Al}-4$ wt pct $\mathrm{Cu}$, it is reasonable to assume that they are also valid for Al-1 wt pct $\mathrm{Cu}$ because the actual $g_{s}(T)$ (or in fact $g_{s}^{\varepsilon}(T$ ) based on the $g_{s}^{0}(T)$ curve given in Figure 5) is used in this case. $^{[21]}$

Finally, the porosity calculation was conducted, using two different estimations of the amount of hydrogen dissolved in the melt $\left([\mathrm{H}]_{0}=0.25\right.$ or $\left.0.4 \mathrm{cc}_{\mathrm{STP}} / 100 \mathrm{~g}\right)$. A constant grain size, $\phi=200 \mu \mathrm{m}$, was used together with standard parameters given elsewhere. ${ }^{[23]}$

Both the thermal and porosity calculations were conducted on an INTEL ${ }^{\ddagger}$ Xeon at $2 \mathrm{GHz}$. The temper-

${ }^{\dagger}$ INTEL is a trademark of Intel Corporation, Santa Clara, CA.

ature field was simulated using elements of approximately $4 \mathrm{~mm}$ in size and time-steps of 2 seconds. The CPU time was 15 minutes, corresponding to $500 \mathrm{sec}-$ onds of physical time. The pore fraction and liquid pressure were calculated using cells of $0.5 \mathrm{~mm}$ and a time-step of 0.01 seconds during the tensile test and 0.1 seconds before. The CPU time was 2 hours. The mechanical problem was solved on an INTEL Itanium 2 at $1.6 \mathrm{GHz}$ with elements $4 \mathrm{~mm}$ in size and an average time-step of typically $10^{-4}$ seconds (automatic time incrementation with the parameter CETOL $=10^{-6}$ in Abaqus ${ }^{[17]}$ ). To simulate 0.5 seconds of an actual tensile test, the CPU time was 2 hours.

\section{RESULTS}

\section{A. Experimental Observations}

Tear tests have been performed in the temperature range of $590{ }^{\circ} \mathrm{C}<T_{\text {tear }}<640{ }^{\circ} \mathrm{C}$. The macroscopic shape of the torn surface is shown in Figure 6 for three different tearing temperatures. In all cases, a fracture occurs at the upper edge of the neck, which is characterized by a slightly higher temperature, i.e., lower $g_{s}$. At $T_{\text {tear }}=640{ }^{\circ} \mathrm{C}$, the fracture surface is flat with a thin needle between the two parts of the casting. This indicates that liquid could still flow fairly easily in between the grains at that temperature. At $T_{\text {tear }}=620{ }^{\circ} \mathrm{C}$, the torn surface is slightly conical, extending a tip toward the upper part, indicating that the crack follows the weakest path, i.e., lowest $g_{s}$ connecting the extremities of the neck. At this critical volume fraction of solid, not all the grain boundaries have bridged and the crack propagates through wet-grain boundaries. At $T_{\text {tear }}=590^{\circ} \mathrm{C}$, most 
Table IV. Summary of the Parameters Involved in the Mechanical Calculation

Mechanical Boundary Condition as Defined in Figure 4

1: axial symmetry

2: bottom

3: bottom half of the mold

4: vertical part of the neck

5: moving half of the mold

6: top surface

Properties of the Al-1 Wt Pct Cu Sample

Young's modulus

Poisson's ratio fixed in the radial direction $\left(u_{r}=0\right)$ and free to move in the longitudinal direction $\left(F_{z}=0\right)$

$u_{z}=0$ and $F_{r}=0$ (no friction assumed in the horizontal plane)

$u_{z}=0$ and $F_{r}=0$

$F_{r}=F_{z}=0$ (note: other conditions in this region did not significantly change the results)

$\dot{u}_{z}=3.3 \mathrm{~mm} \mathrm{~s}^{-1}$ and $F_{r}=0$

$F_{r}=F_{z}=0$

$\mathrm{E}=70 \mathrm{GPa}$ at $20{ }^{\circ} \mathrm{C}, \mathrm{E}=40 \mathrm{GPa}$ at $500{ }^{\circ} \mathrm{C}$ and decreases to $\mathrm{E}=0.04 \mathrm{GPa}$ for the mushy alloy

$v=0.33$

Table V. Parameters Describing the Rheology of the Al-1 Wt Pet Cu Alloy

\begin{tabular}{llllll}
\hline$s_{0}(\mathrm{MPa})$ & $A\left(\mathrm{~s}^{-1}\right)$ & $Q\left(\mathrm{kJmol}^{-1}\right)$ & $n$ & & \\
4.77 & $910^{5}$ & 154 & 3.8 & & \\
$P$ & $\alpha_{0}$ & $\alpha_{1}$ & $g_{s}^{\text {coal }}$ & $k$ \\
0.11 & 4.45 & $1.07 \cdot 10^{-2}$ & 0.95 & $g_{s}^{\text {coh }}$ & 0.65 \\
\hline
\end{tabular}

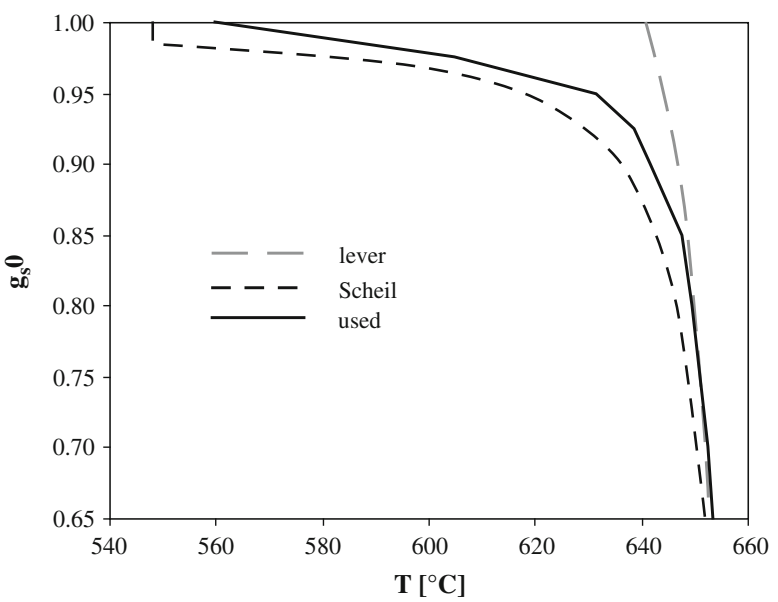

Fig. 5-Solidification path of the Al-1 wt pet $\mathrm{Cu}$ alloy.
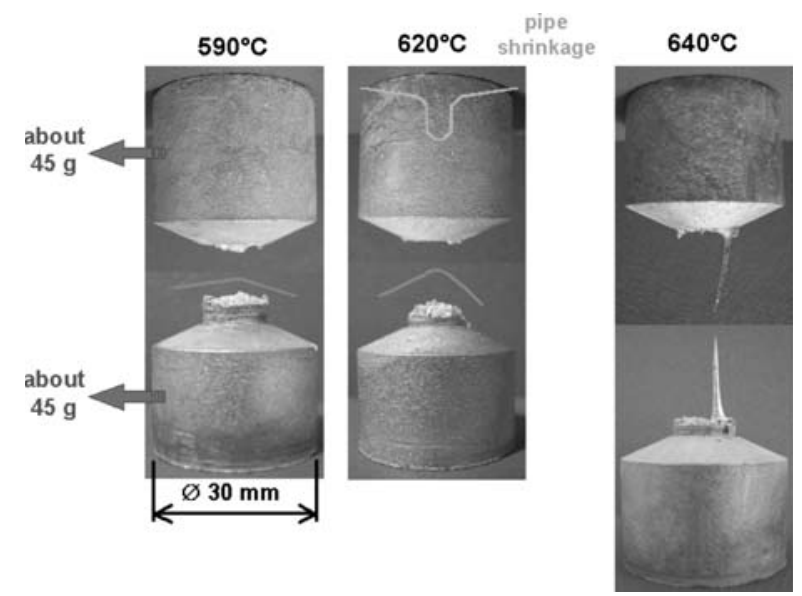

Fig. 6-Macroscopic tear surface profiles of samples torn at different temperatures $T_{\text {tear }}$. of the grain boundaries have bridged, and the surface of the crack becomes flatter.

After the tensile test, the ruptured surfaces were observed by scanning electron microscope (SEM). The macroscopic shape of the crack surface could be correlated with its microscopic features. For $T_{\text {tear }}=590{ }^{\circ} \mathrm{C}$ (Figure 7), the crack surface is particularly damaged but fairly flat. In Figure 8(a), the circles highlight a few zones where the smooth structure of the dendrite arms can still be observed, whereas in Figure 8(b), they show spikes present at the surface. In these regions, there must have been thin liquid films remaining at the moment of tearing. During tearing, liquid menisci can form between two solid surfaces, separating still wet-grain boundaries; these menisci form solid spikes after complete solidification. ${ }^{[12]}$ The arrows in Figure 7 indicate some broken spots with sharp-edge shapes, where coalescence was already well advanced. In Figure $8\left(\right.$ a) $\left(T_{\text {tear }}=620^{\circ} \mathrm{C}\right)$, the surface is much smoother and strictly follows the liquid-grain boundaries, indicating the presence of liquid films at the time of fracture. Many spikes can be also observed on the surface (circles), but a few quite large sharp-edge zones can also be observed (arrows). Finally, observing a sample broken at $T_{\text {tear }}=630^{\circ} \mathrm{C}$ (Figure 8(b)), a completely smooth surface perfectly following the shape of the grain boundaries and dendrite arms can be observed, with a few spikes (circles). However, the arrows highlight several coalescence "lines" that were broken during tearing (a coalescence line starts as a coalescence bridge between two grains/dendrites, which then extends between two surfaces as a line). The square in Figure 8(b) shows a solid bridge that was not broken during tearing.

\section{B. Model Validation}

The thermal field predicted in the sample is illustrated in Figure 9. Solidification progresses along the axial direction, and the isotherms are perpendicular to the 


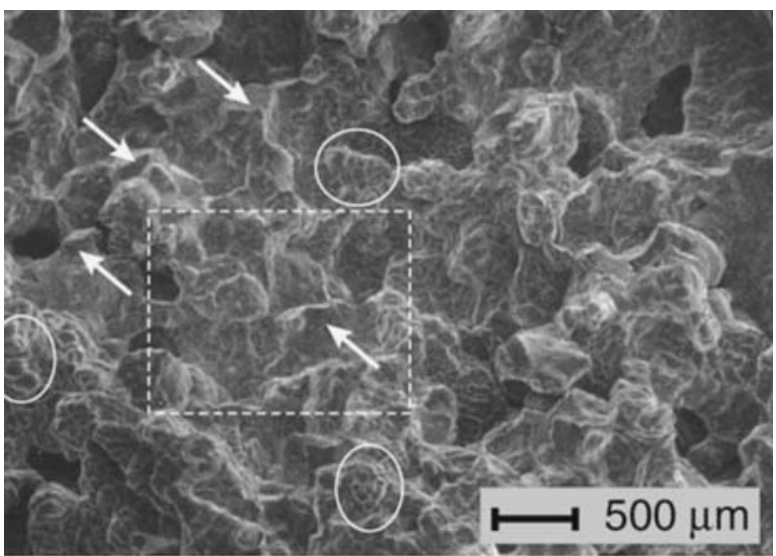

(a)

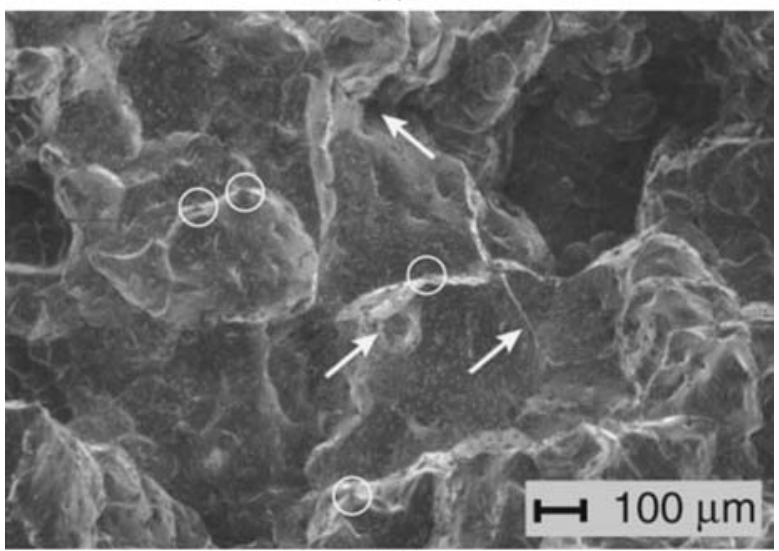

(b)

Fig. 7-SEM micrographs of an $\mathrm{Al}-1$ wt pet $\mathrm{Cu}$ fracture surface obtained by tearing at $T_{\text {tear }}=590{ }^{\circ} \mathrm{C}$ for two magnifications.

principal loading axis in the neck region. In order to assess the validity of this model, the simulated value of the temperature at the location where the thermocouple is inserted is compared to the experimentally measured values (corresponding to different experiments) (Figure 10). The experimental cooling conditions are well reproduced from one test to another, with most of the latent heat released close to the liquidus in this lowconcentration alloy. A nearly constant cooling rate of $-0.4 \mathrm{Ks}^{-1}$ is obtained in the coherent-mushy zone before tearing occurs (at the times indicated with a cross on the gray experimental curves). Once the upper mold starts to move, the measured temperature rapidly decreases. Except for this last feature, which is not accounted for in the thermal calculation, the simulated cooling curve exhibits the same features and represents accurately the evolution of the temperature field in the sample. This simulated-temperature field will be used as an input for the mechanical and porosity calculations, as well as for the interpretation of the results. For example, the simulation gives an estimation of the vertical thermal gradient in the neck region. This gradient was approximately $5 \mathrm{Kmm}^{-1}$ under these conditions.

The validity of the mechanical model was then investigated by comparing the experimental tensile curves (force $F=F_{z} v s$ displacement $u=u_{z}$ ) and the

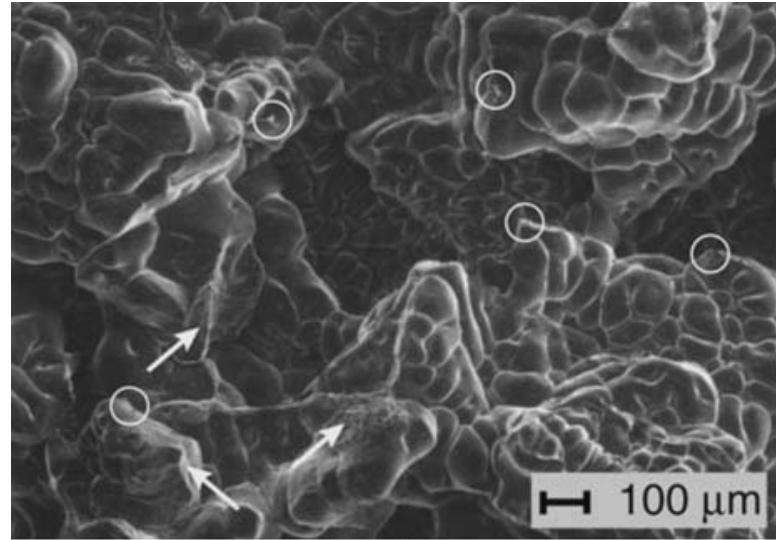

(a)

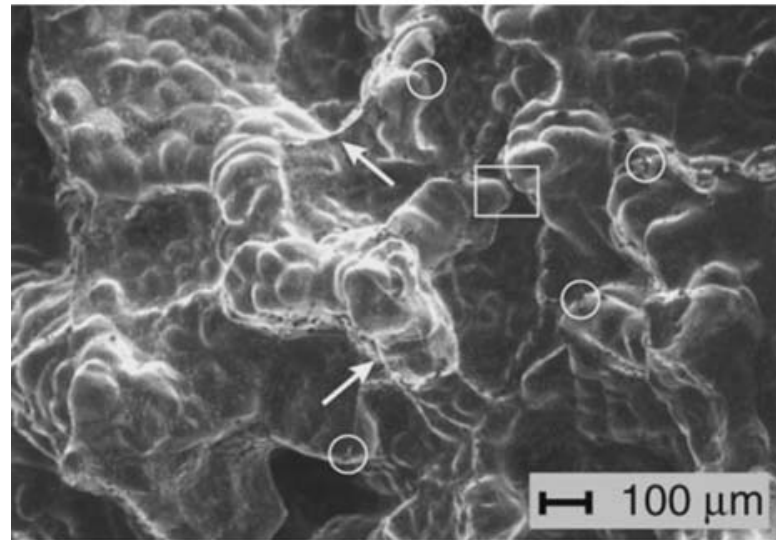

(b)

Fig. 8-SEM micrographs of $\mathrm{Al}-1$ wt pct $\mathrm{Cu}$ fracture surfaces obtained by tearing at (a) $T_{\text {tear }}=620{ }^{\circ} \mathrm{C}$ and $(b) T_{\text {tear }}=630{ }^{\circ} \mathrm{C}$.

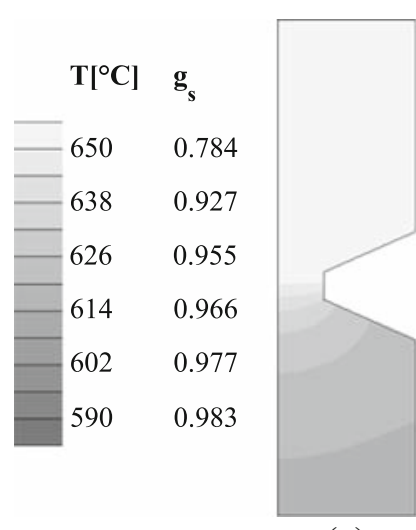

(a) (b)

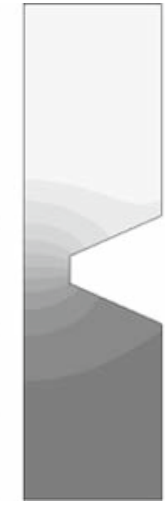

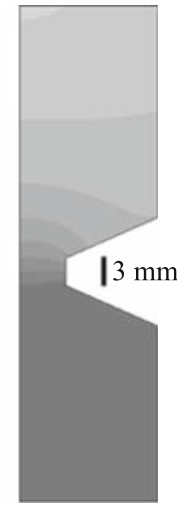

(c)
Fig. 9-Thermal field simulated in the sample at three different times: (a) $205 \mathrm{~s}$, (b) $255 \mathrm{~s}$, and (c) $305 \mathrm{~s}$. The temperature range in which the tensile tests were conducted is displayed in detail. Please note that the corresponding solid fraction is also indicated on the temperature scale.

simulated ones. The results obtained for the tests made in the upper and lower temperature ranges are shown in Figure 11 and Figure 12, respectively. The agreement between the actual measurements and the simulations is very good, especially for the tests carried out at the 

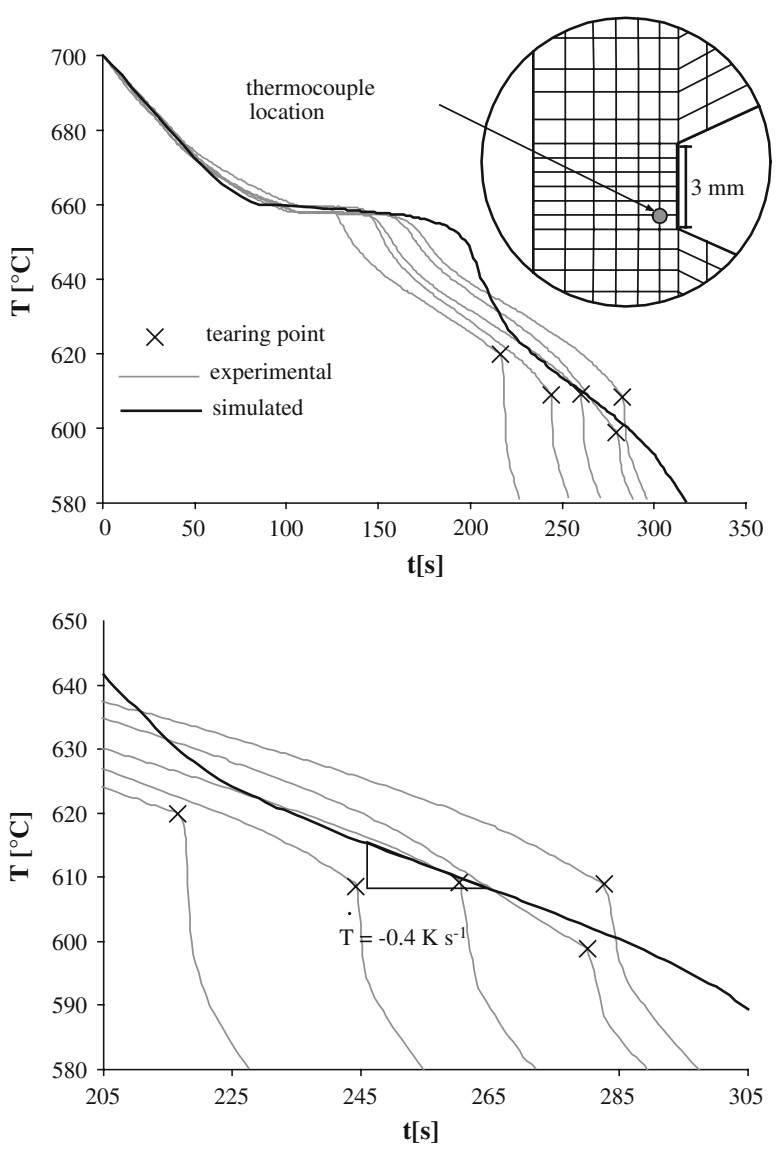

Fig. 10-Position of the thermocouple and comparison between experimental temperature measurements (thin gray lines, corresponding to different tests representing the experimental scatter) and the result given by the simulation (thick black line). Please note that the point at which tearing occurred is indicated by a cross on the experimental curves.

lowest temperatures, i.e., highest solid fractions (Figure 12). Of course, the mechanical model does not predict fracture, i.e., the rapidly decreasing part of the curves, $F(u)$. However, before this event, the calculated and simulated curves almost coincide. A slightly larger discrepancy is observed in the tests carried out at the highest temperatures, i.e., lowest solid fractions (Figure 11). There are two reasons for this: (1) the forces measured at the highest temperatures are low and any friction might perturb the measurement; and (2) the thermal model predicts a temperature that is slightly overestimated as it gets closer to the liquidus. Both explanations tend to make the measured forces higher than the simulated ones, which are indeed observed for the tearing tests performed at the highest temperatures $\left(635^{\circ} \mathrm{C}\right.$ and $640{ }^{\circ} \mathrm{C}$ ). Based on this agreement, the thermomechanical model may thus be used for more advanced characterization of the results, as seen in the following section.

At this stage, it is worth making a few comments about the maximum force found in both the experimental and simulated tensile curves. Clearly, the maximum force measured in the experiment corresponds to failure (tearing) of the sample. As far as the simulation is

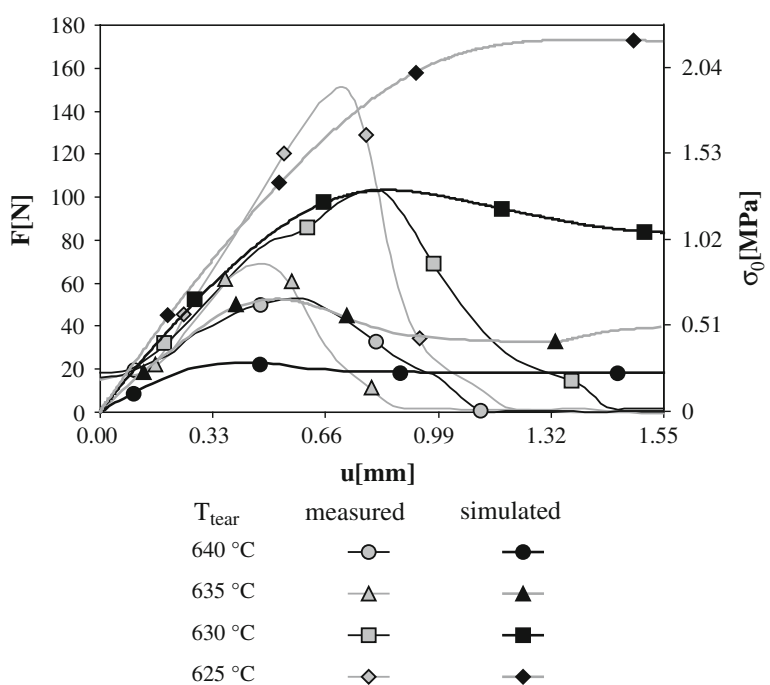

Fig. 11-Comparison between the measured and simulated reaction forces for different values of $T_{\text {tear }}$. The nominal stress, $\sigma_{0}$, corresponding to $F$ was computed from the cross-sectional area at the neck and is shown on the alternative scale on the right. Curves with the same line shade and symbol shape are to be compared.

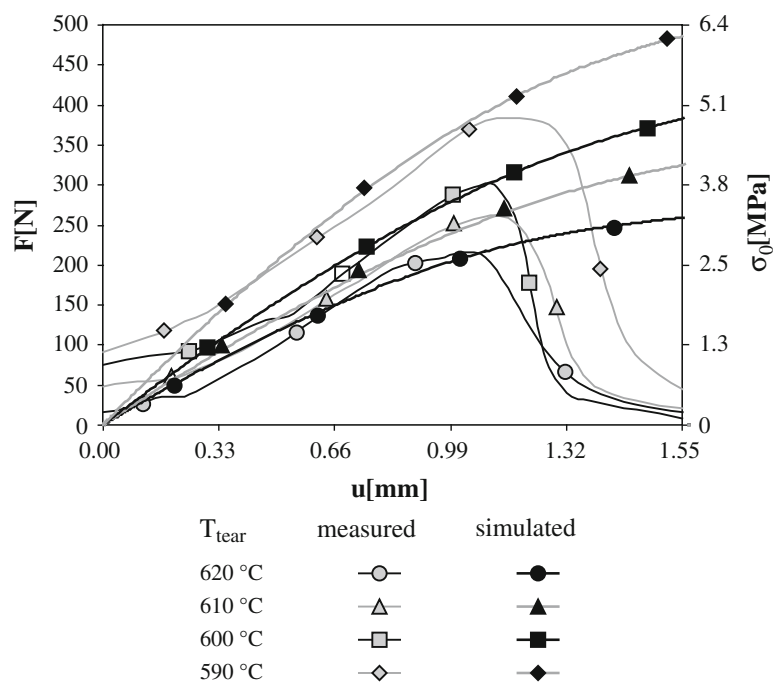

Fig. 12 - Comparison between the measured and simulated reaction forces for different values of $T_{\text {tear }}$. The nominal stress, $\sigma_{0}$, corresponding to $F$ was computed from the cross-sectional area at the neck and is shown on the alternative scale on the right. Curves with the same line shade and symbol shape are to be compared.

concerned, it is not obvious why a maximum force is found for the highest temperature tests, since, as stated before, no failure model is included in this calculation. It turns out that stresses and strains tend to be concentrated at the top of the neck and close to the mold. The concentration of strain in the neck of the sample, as illustrated in Figure 13, is obtained from the mechanical simulation. It should be noted that the strain distribution is quite inhomogeneous due to the sample geometry (most of the strain is concentrated in the neck region). Typical values of the axial strain, corresponding to 


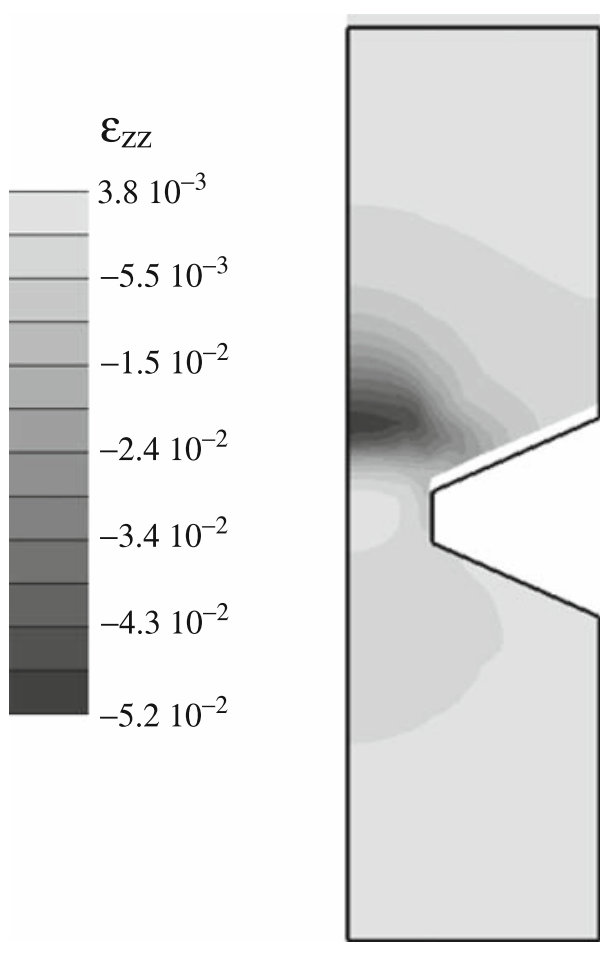

Fig. 13-Axial strain field represented on the deformed shape (superimposed on the undeformed sample shape in thick black line). This image corresponds to a displacement of the top of the mold of $u_{z}=$ $0.83 \mathrm{~mm}(t=0.25 \mathrm{~s})$ and to a tearing temperature $T_{\text {tear }}=630^{\circ} \mathrm{C}$.

tearing of the sample, range from a few tenths of percent to a few percent.

After some amount of deformation, the cohesion of the mushy alloy begins decreasing due to the large applied strain rate. Therefore, the local solid fraction, $g_{s}(T)$, decreases due to the volumetric strain, thus reducing the $C^{*}$ value and, consequently, the cohesion parameter, $C$. This decohesion will cause the strength of the material to decrease locally, thus explaining the maximum observed in the simulated tensile curves. We shall come back to this observation in the following section.

\section{DISCUSSION OF RESULTS}

The maximum force recorded during the experimental tensile test, $F_{\text {fail }}$, is proportional to the tensile strength, $\sigma_{\text {fail }}$, of the mushy alloy, which increases with the solid fraction. In order to accurately describe this behavior, it is necessary to use information from both the experiments and the numerical model. Indeed, the thermocouple inserted in the sample does not measure the temperature at the actual location of the tear. As a consequence, the displacement at failure and the position of the crack front are extracted from the experimental results. From this information, the results of the simulations can be used to determine the actual solid fraction at the location of the crack. Please note that the temperature difference between the thermocouple position and the position of the crack is quite large due to the vertical thermal gradient (i.e., a difference in the neck height of $3 \mathrm{~mm}$ induces a temperature difference of $\left.15^{\circ} \mathrm{C}\right)$. Considering the steep part of the $g_{s}(T)$ curve near the liquidus (Figure 5), this significantly corrects the value of $g_{s}$ for the tearing tests performed at highest temperatures (i.e., from $g_{s}=0.92$ as approximated from experimental temperature records to $g_{s}=0.5$ as estimated more accurately by using simulation results). This effect is reduced for the tests performed at the lowest temperatures, since $g_{s}(T)$ is much flatter. It is nevertheless important since the mechanical behavior of the mushy zone changes drastically over a very narrow $g_{s}$ interval (Figure 14). In this figure, the tensile strength of the material is displayed as a function of the value of $g_{s}^{\varepsilon}$ estimated at the crack position, including the swelling contribution. Such a representation is helpful to distinguish between three different regions (ranges of actual solid fraction $g_{s}^{\varepsilon}$ ), which can be correlated with the observations mentioned in Section IV.

(1) For $0.5<g_{s}<\sim 0.9$, the resistance of the mushy zone is fairly weak but increases steadily with $g_{s}$. The globulodendritic grains already occupy most of the volume with liquid films remaining in between. Resistance of the mushy zone is mainly achieved by reduced mobility of the grains and dendrites. Some solid bridges are likely already established among dendrite arms within the grains and at very few grain boundaries, where the misorientation is low. In this region, although the mushy zone does not offer much resistance, ductility can be achieved by easy feeding. As deformation occurs, fluid flow compensates any opening occurring at grain boundaries. This is clearly visible in Figure 6 for the test performed at $640{ }^{\circ} \mathrm{C}$, where an outflow is observed on the top part of the casting.

(2) For $\sim 0.9<g_{s}<\sim 0.94$, a fairly sharp increase of the maximum stress can be observed in Figure 14. This likely corresponds to the beginning of percolation of the grains with the gradual disappearance of liquid films at the boundaries. A crack will try to follow a connected path among grain boundaries that are still liquid, thus explaining the curved shape of the torn surface in Figure 6. This region, outlined in gray in Figure 14, corresponds to the "brittle" region of the solidification interval; the resistance offered to strain of the mushy zone is still low, but feeding is no longer possible.

(3) For $\sim 0.94<g_{s}$, most of the liquid films have been replaced by solid bridges with some remaining liquid droplets, and deformation occurs mainly within a viscoplastic continuous solid. The crack surface is flatter, and the stress at fracture steadily increases. Ductility in this case is achieved by plastic deformation as in a fully coalesced solid material.

The solid fraction value, at which an increase of the mechanical strength takes place, is in agreement with the literature. ${ }^{[15,16]}$ It can also be put into relation with observations made in organic-alloy solidification. ${ }^{[12]}$ 


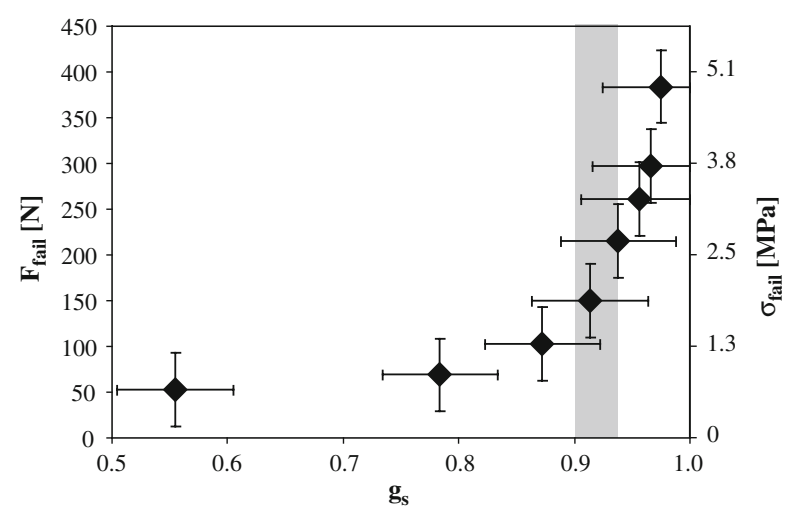

Fig. 14-Maximum force (tensile strength) of the mushy alloy as a function of the solid fraction when and where tearing actually occurred.

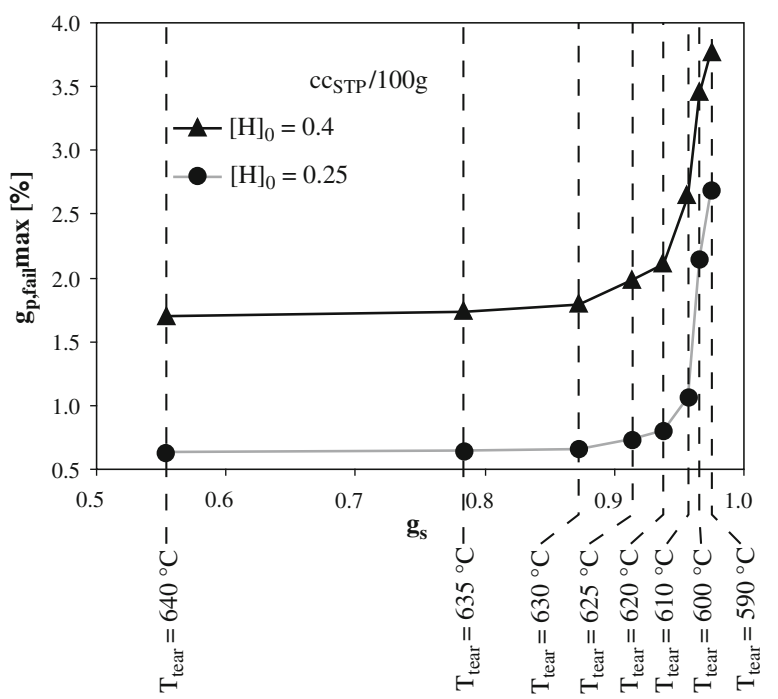

Fig. 15-Maximum porosity fraction in the sample as a function of the solid fraction at the location where tearing occurred. Please note that the error bars on $g_{s}$ are not displayed for clarity but are identical to those in Fig. 14.

Besides the results of the thermal and mechanical calculations, it is also interesting to examine the predictions of the porosity module (Figure 15). When tearing occurs at a rather low solid fraction $\left(g_{s}<0.9\right)$, the volume fraction of porosity, $g_{p}$, remains fairly constant, regardless of the swelling experienced by the mush during tearing. This can be explained by the fact that micropores form mainly as a result of segregation of hydrogen in the liquid phase, feeding being easy at such low volume fraction of solid. Increasing the hydrogen content $[\mathrm{H}]_{0}$ from 0.25 to $0.4 \mathrm{cc}_{\mathrm{STP}} / 100 \mathrm{~g}$ nearly triples the fraction of porosity. Please note that the amount of porosity is a nonlinear function of the gas concentration as a minimal amount is necessary for the melt to be supersaturated. In this regime, the mechanical model also predicts decohesion (solid grains are pulled apart due to strain, and the strength of the material decreases) close to the displacement where tearing is observed experimentally. Failure is thus related to an unstablestrain-concentration phenomenon and not to the nucleation and growth of a void (defect).
On the other hand, when $g_{s}>0.94$, the amount of porosity found in the neck of the sample increases rapidly with $g_{s}$. In this regime, the porosity fraction, $g_{p}$, is mainly due to the fact that the rate of swelling of the mushy zone is high, due to the rather high value that was selected for the imposed displacement rate. This means that significant voiding (damage) develops due to strain in the sample before it actually breaks. Under such conditions, tearing (in the experiment) clearly occurs before decohesion (as predicted by the numerical model). In this case, failure thus occurs by a damaging mechanism. Between this damaging regime and the low-solid-fraction behavior described in a preceding section, the brittle zone (where hot tearing is likely to happen) corresponds to a transition in the fracture mode of the mushy alloy.

In summary, the experimental tensile tests, SEM observations, and the results of the numerical model can all be correlated. Below 90 pct of solid, the hot tear progresses easily through the continuous network of intergranular liquid films. Failure is initiated due to a mechanical instability (decohesion predicted by the mechanical model) without prior damage of the material. Above 94 pet of solid, which is typically the value that this study indicates as the coalescence point of the Al-1 wt pet $\mathrm{Cu}$ alloy, the tear can only advance if solid bridges are deformed and broken. Failure occurs when the applied strain exceeds the ductility of the material and damaging of the sample develops before failure. Between typically 90 and 94 pct of solid, the behavior of the mushy alloy continuously varies between the two limiting cases exposed previously. The ability of the alloy to withstand strain is expected to be minimal in that range of solid fraction, corresponding to the highest hot-tearing susceptibility.

\section{CONCLUSIONS}

The present article presents a new setup designed to perform tensile tests on mushy alloys. This experiment can be used to study coalescence in low-concentration alloys in the context of hot-tearing predictions. The measurements performed using this setup can be interpreted in detail thanks to complementary information available from the numerical model established to represent the casting. These two aspects are used to study the development of a continuous network of solid at a late stage of solidification. The results from tensile-strength measurement, fracture-surface observations, thermalfield prediction, mechanical simulation, and porosityformation pattern clearly establish that there is a drastic change in the fracture mode of the alloy in the range 90 to 94 pct volume fraction of solid. This transition corresponds to the percolation of the grains into a coherent network by coalescence, which can thus be clearly measured and described using the present method.

\section{ACKNOWLEDGMENTS}

The authors thank the European Community within the framework of the VIRCAST project (Contract No. 
G5RD-CT-2000-00153) for funding the experimental aspects. The authors also thank J.-D. Wagnière and Dr. L. Weber for setting up the experiment. As far as modeling is concerned, the work was carried out as part of the POST project. The authors also thank the Commission for Technology and Innovation (CTI, Grant No. 6167.1), as well as the industrial partners, Alcan (CH and FR), HydroAluminium (DE), Umicore (BE), and General Motors (United States), for their financial support. Finally, the authors thank Drs. G. Couturier, J.-M. Drezet, and O. Ludwig for their help with the porosity module and mechanical models, as well as J.-L. Desbiolles for his assistance on software aspects.

\section{REFERENCES}

1. D.G. Eskin, Suyitno, and L. Katgerman.: Progr. Mater. Sci., 2004, vol. 49 , pp. 629-711.

2. U. Feurer: Giessereiforschung, 1976, vol. 28, pp. 750-80.

3. T.W. Clyne and G.J. Davies: J. Br. Foundry, 1981, vol. 74, pp. 65-73.

4. M. Rappaz, J.-M. Drezet, and M. Gremaud: Metall. Mater. Trans. A, 1999, vol. 30A, pp. 449-55.

5. M. Rappaz, A. Jacot, and W.J. Boettinger: Metall. Mater. Trans. A, 2003, vol. 34A, pp. 467-79.

6. V. Mathier, M. Rappaz, and A. Jacot: Modell. Simul. Mater. Sci. Eng., 2004, vol. 12, pp. 479-90.

7. S. Vernède and M. Rappaz: Philos. Mag., 2006, vol. 86, pp. 3779 94.
8. T.W. Clyne and G.J. Davies: J. Br. Foundry, 1975, vol. 68, pp. 238-44.

9. J.A. Spittle and A.A. Cushway: Met. Technol., 1983, vol. 10, pp. 6-13.

10. D. Warrington and D.G. McCartney: Cast Met., 1989, vol. 2, pp. 134-43.

11. P. Ackermann, W. Kurz, and W. Heinemann: Mater. Sci. Eng., 1985, vol. 75, pp. 79-86.

12. I. Farup, J.-M. Drezet, and M. Rappaz: Acta Mater., 2001, vol. 49 , pp. 1261-69.

13. S. Vernède, P. Jarry, and M. Rappaz: Acta Mater., 2006, vol. 54, pp. 4023-34

14. B. Magnin, L. Maenner, L. Katgerman, and S. Engler: Mater. Sci. Forum, 1996, pp. 217-22 and pp. 1209-14.

15. Y. Ju and L. Arnberg: Int. J. Cast Met. Res., 2003, vol. 16, pp. $522-30$.

16. O. Ludwig, J.-M. Drezet, C.L. Martin, and M. Suéry: Metall. Mater. Trans. A, 2005, vol. 36A, pp. 1525-35.

17. V. Mathier, J.-M. Drezet, and M. Rappaz: Modell. Simul. Mater. Sci. Eng., 2007, vol. 15, pp. 121-34.

18. V. Mathier: Ph.D. Dissertation, Ecole Polytechnique Fédérale de Lausanne, Lausanne, Switzerland, 2007.

19. M. M'Hamdi, A. Mo, and H.G. Fjaer: Metall. Mater. Trans. A, 2006, vol. 37A, pp. 3069-83.

20. V. Mathier, J.-M. Drezet, and M. Rappaz: MCWASP XI, 2006.

21. O. Ludwig, J.-M. Drezet, P. Ménès, C.L. Martin, and M. Suéry: Mater. Sci. Eng. A, 2005, vols. 413-414, pp. 174-79.

22. W.M. Van Haaften, B. Magnin, W.H. Kool, and L. Katgerman: Metall. Mater. Trans. A, 2002, vol. 33A, pp. 1971-80.

23. C. Pequet, M. Gremaud, and M. Rappaz: Metall. Mater. Trans. A, 2002, vol. 33A, pp. 2095-2106.

24. P.-D. Grasso: Ph.D. Dissertation, Ecole Polytechnique Fédérale de Lausanne, Lausanne, Switzerland, 2004. 